\title{
Hepatitis B surveillance in Canada: 2005-2012
}

\author{
Payne $\mathrm{E}^{1^{\star}}$, Totten $\mathrm{S}^{1}$, Laroche $\mathrm{J}^{2}$ and Archibald $\mathrm{C}^{1}$ \\ 1 Public Health Agency of Canada, Centre for Communicable Diseases and Infection Control, Ottawa, ON \\ 2 Public Health Agency of Canada, Centre for Immunization and Respiratory Infectious Diseases, Ottawa, ON \\ * Corresponding author: elspeth.payne@phac-aspc.gc.ca
}

\section{Abstract}

Objective: To describe surveillance trends by age, sex and infection status of Hepatitis B (HB) virus in Canada between 2005 and 2012.

Methods: Data on acute and chronic HB cases reported to the Canadian Notifiable Disease Surveillance System were compiled at the national level to examine trends by age and sex over time. Time trends are presented from 2005 to 2012, corresponding to the availability of data differentiated by acute and chronic infection status.

Results: The rate of reported acute HB infections decreased from 1.0 to 0.6 per 100,000 between 2005 and 2012. Both sexes showed rate decreases over this time, although acute HB rates were consistently higher among males than females. The rate of reported chronic HB infections decreased from 14.1 to 12.0 per 100,000 between 2009 and 2012. These results are based on data in which infection status was specified. The proportion of unspecified cases in any given year may somewhat alter the results.

Conclusion: This is the first time that trends in reported cases and corresponding rates of HB in Canada have been examined by acute and chronic infection status using national surveillance data. Canada continues to have a downward trend in HB rates across Canada, most notably in acute HB cases. Increasing national capacity to differentiate between acute and chronic $\mathrm{HB}$ will facilitate a more thorough understanding of trends in transmission and of the burden of HB infection in Canada.

\section{Introduction}

Hepatitis $B(\mathrm{HB})$ is an important clinical and public health issue worldwide. Globally, it is estimated that two billion people have been infected with HB virus (1) and approximately 400 million individuals are chronically infected carriers (2). Approximately $10 \%$ of infants infected at birth and over $90 \%$ of adults will recover completely from $\mathrm{HB}$; in the rest, chronic HB infection develops, which may result in severe illness and premature death (3). In 2011, acute HB infection was reported as the underlying cause of 43 deaths in Canada while chronic HB infection was reported as the underlying cause of 19 deaths (4). This may be an underestimate, as deaths attributable to chronic HB may have been coded as more proximal causes such as hepatocellular carcinoma or cirrhosis.

The rates of acute HB observed in Canada are low, and routine HB vaccination programs have undoubtedly contributed to this low rate. In Canada, all provinces and territories have had an HB vaccination program since the 1990s (5). Although programs differ by jurisdiction, all offer HB immunization to infants and/or school-aged children (6) and in some jurisdictions to individuals at increased risk $(7,8)$. In 2009, HB immunization coverage by the second birthday was estimated to be $69 \%$ in provinces and territories with a three-dose infant program (9). Coverage with at least two doses of the HB vaccine by the $17^{\text {th }}$ birthday was $74.8 \%$ in $2011(10)$. In 2012 , national HB immunization coverage was estimated at only $39.7 \%$ in the non-institutionalized adult population; however, approximately $64.9 \%$ of health care workers in close contact with patients had received the HB vaccine (11).

Diagnosis of HB requires laboratory confirmation by means of a blood sample to differentiate HB infection from other types of hepatitis. Infection markers present in the blood can also be used to distinguish between acute and 
chronic HB infection. Until recently, surveillance data reported to the Canadian Notifiable Disease Surveillance System (CNDSS) by most provinces and territories did not distinguish between acute and chronic HB infection, starting with data reported for 2005 , a number of provinces and territories began reporting $\mathrm{HB}$ cases differentiated by acute and chronic infection status. The objective of this analysis was to describe surveillance trends by age, sex and infection status in Canada between 2005 and 2012.

\section{Methods}

\section{Data sources}

HB has been nationally notifiable since 1969; data on acute and chronic HB cases are reported to the CNDSS by provincial and territorial ministries of health, which in turn obtain data from local and regional health authorities. Most provinces and territories distinguish reported $\mathrm{HB}$ cases by infection status, namely acute, chronic and unspecified HB infection (refer to Table 1 for case definitions). Reporting of infection status by provinces and territories has increased over time; however, some cases are still reported without infection status. Only acute and chronic HB cases are analyzed in detail in this report, thus presented results are not inclusive of all HB cases reported to the CNDSS.

Table 1. Hepatitis B case definitions used under the CNDSS

\begin{tabular}{l|l}
\hline Infection status & Case definition (12) \\
\hline Acute HB infection & $\begin{array}{l}\text { HBsAg and anti-HBc IgM positive in the context of a compatible clinical } \\
\text { history or probable exposure } \\
\text { or } \\
\text { clearance of HBsAg in a person who was documented to be HBsAg positive } \\
\text { within the last six months in the context of a compatible clinical history or } \\
\text { probable exposure. }\end{array}$ \\
\hline Confirmed chronic HB infection & $\begin{array}{l}\text { A person being HBsAg positive for more than } 6 \text { months } \\
\text { or } \\
\text { detection of HBsAg in the documented absence of anti-HBc IgM } \\
\text { or } \\
\text { detection of HB DNA for more than } 6 \text { months. }\end{array}$ \\
\hline Unspecified HB infection & $\begin{array}{l}\text { Serologic profile not in line with either acute or chronic case definition and } \\
\text { HBsAg positive } \\
\text { or } \\
\text { detection of HB DNA. }\end{array}$ \\
\hline
\end{tabular}

* For the purposes of this report, cases reported as unspecified could also include cases not differentiated as acute or chronic by the reporting province or territory. HBsAG: Hepatitis B surface antigen. anti-HBc IgM: IgM antibody against Hepatitis B core antigen.

\section{Analysis}

Descriptive analysis of HB infection by year, age group and sex was conducted using data reported to the CNDSS. Analyses are presented separated by acute and chronic HB infection status, and rates are given per 100,000 population. Rates, percentages, and percent change in rates were calculated using unrounded numbers, thus presented rounded numbers may not sum to the total. Population data for the calculation of rates were provided by Statistics Canada, Demography Division, Demographic Estimates Section. The following estimates were used: for 2005, final intercensal estimates; for 2006-2009, final postcensal estimates; for 2010-2011, updated postcensal estimates; and for 2012; preliminary postcensal estimates. 
In order to examine trends over time, $\mathrm{HB}$ analyses were restricted to provinces and territories that consistently provided acute and/or chronic HB data to the CNDSS over the entire time frame used. Acute HB analysis for the time period 2005 to 2012 involved data from British Columbia, Alberta, Saskatchewan, Manitoba, Ontario, Quebec, New Brunswick, the Yukon and the Northwest Territories. In some provinces and territories, chronic HB did not become reportable until recent years; it remains non-reportable in Ontario. Reporting of chronic $\mathrm{HB}$ infection became more consistent in 2009; thus, trend analysis of chronic HB was restricted to 2009 to 2012. It includes data from British Columbia, Alberta, Saskatchewan, Quebec, New Brunswick and Nova Scotia. Two provinces (Newfoundland and Labrador and Prince Edward Island) provided exclusively unspecified HB data over the entire reporting period and thus are not reflected in any of the national-level analyses presented here.

Demographic patterns (age and sex) were examined for cases of HB reported in 2012 to provide a more detailed snapshot of the most recent available data. In these analyses, all provinces and territories that reported HB cases for that year were included; thus, in 2012, data on acute HB cases from Nova Scotia and on chronic HB cases from Manitoba and Yukon were also included.

\section{Results}

\section{Acute HB infection}

\section{Trends over time}

The total rate of reported cases of acute HB infection decreased steadily between 2005 and 2012. In 2005, a total of 304 cases of acute HB infection were reported, corresponding to an overall rate of 1.0 per 100,000. In 2012, 183 cases were reported, which represents a rate of 0.6 per 100,000 (Figure 1).

Between 2005 and 2012, rates of reported cases of acute HB were consistently higher among males than females, although the gap between the sexes narrowed over time. Though rate decreases were observed over time in both sexes, males showed a greater decrease (53.8\% for males versus $19.8 \%$ for females) (Figure 1).

Figure 1. Reported number of cases and rates of acute HB infection in Canada* by sex, CNDSS, 2005-2012

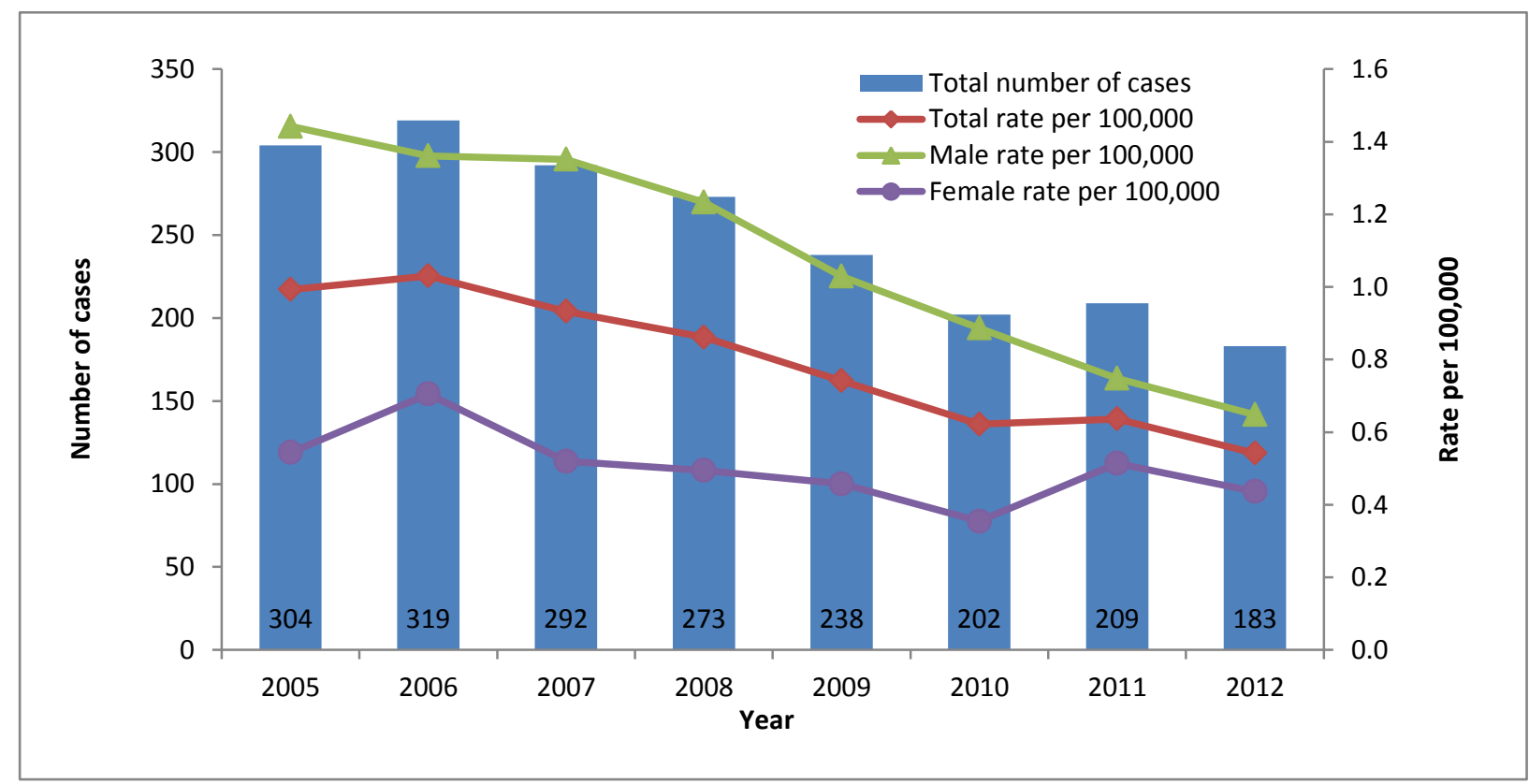

*Includes $B C, A B, S K, M B, O N, Q C, N B, Y T, N T$ 


\section{Trends by age group and sex}

Between 2005 and 2012, the rates of reported cases of acute HB among males decreased across all age groups. There were consistently fewer acute HB cases reported in males less than 25 years of age, corresponding to rates of 1.0 per 100,000 or less in the younger age groups for all years. In 2005 , males aged 30 to 39 years had the highest rate of reported acute HB infection at 3.1 per 100,000. In 2012, this rate had decreased to 1.5 per 100,000. A large decrease in rates was also observed among males aged 40 to 59 during this period, from 2.2 to 0.9 per 100,000 (data not shown).

Among females, both increases and decreases in rates of reported cases of acute HB were observed between 2005 and 2012. Most changes in rates were marginal, with the exception of females in the 20 to 24 age group whose rate decreased by $71.8 \%$, from 1.3 to 0.4 per 100,000 . Few cases were reported among females, especially in the younger age groups (<30 years). Continued monitoring of subsequent years of data will be useful in identifying any emerging trends among females.

In 2012, the highest rate of reported cases of acute HB infection was observed among males in the 30 to 39 age group (1.5 per 100,000), followed by females in the 25 to 29 age group (1.1 per 100,000). Overall, for both sexes rates of acute HB were higher among those aged 25 to 59 years and lower among both younger ( $<20$ years) and older (> 60 years) age groups (Figure 2).

Figure 2. Rates of reported cases of acute HB in Canada* by age group and sex, CNDSS, 2012

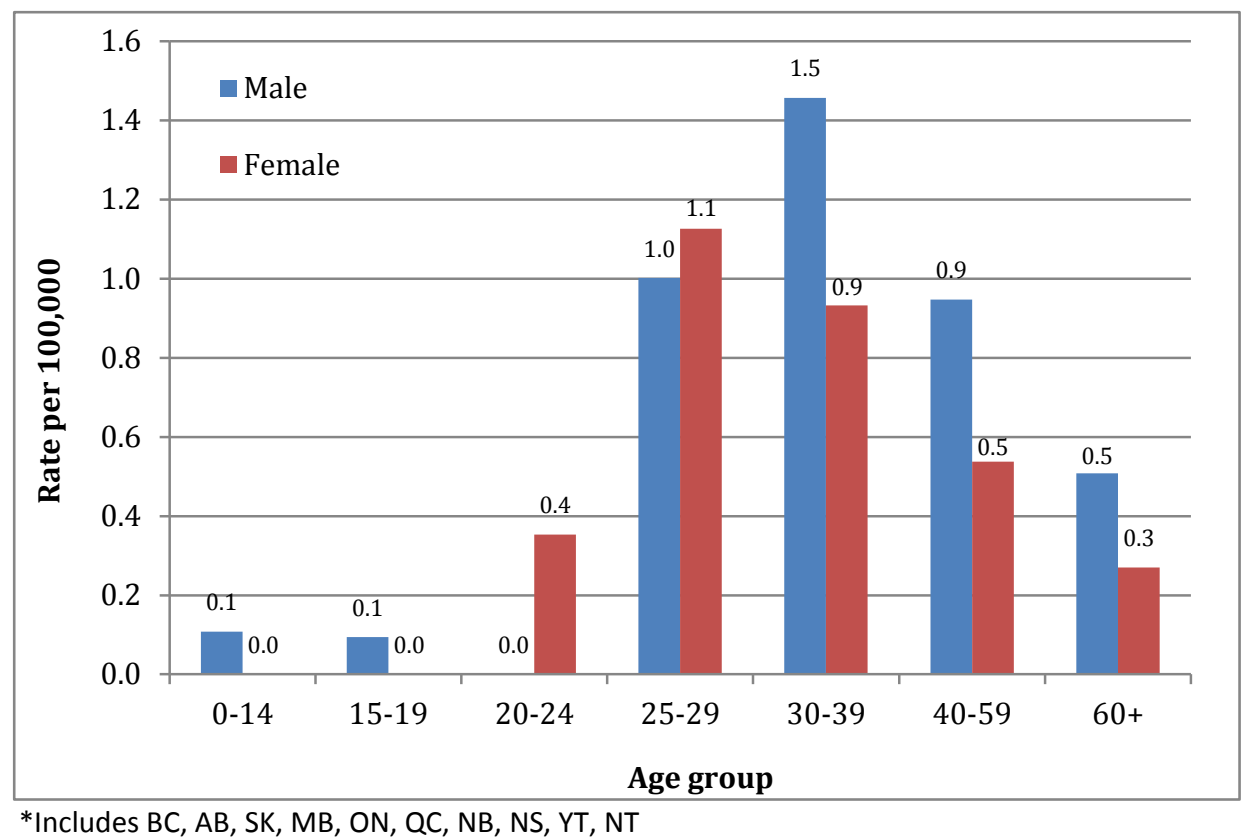

\section{Chronic HB infection}

There was some variation in the rate of reported cases of chronic HB infection between 2009 and 2012, but the overall trend was a decrease over this time frame. In 2009, a total of 2,631 cases of chronic HB were reported, corresponding to an overall chronic HB rate of 14.1 per 100,000. In 2012, there were 2,314 cases, resulting in a rate of 12.0 per 100,000. Between 2009 and 2012, chronic HB rates were consistently higher among males as compared with their female counterparts, though rate increases and decreases were observed in both sexes (Figure 3). 
Figure 3. Reported number of cases and rates of chronic infection in Canada* by sex, CNDSS, 2009-2012

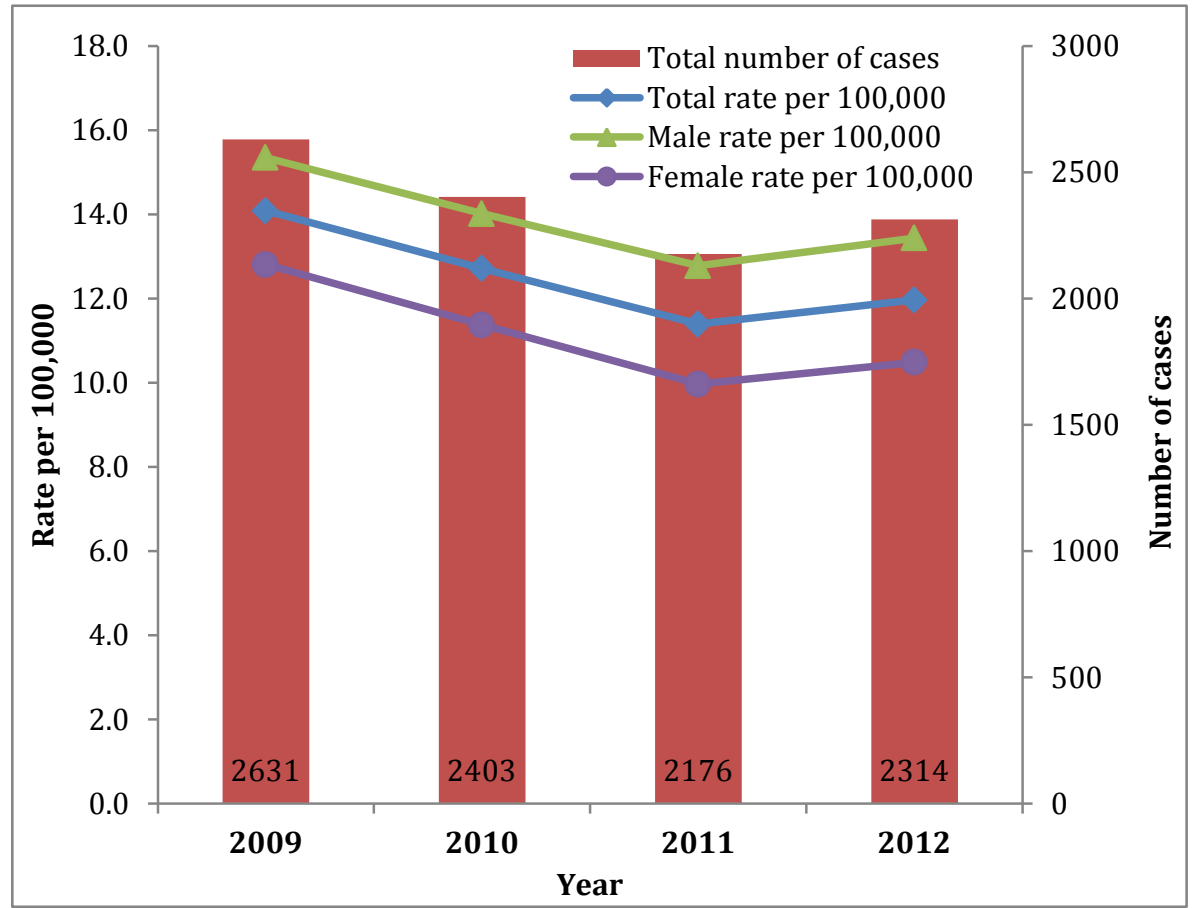

*Includes BC, AB, SK, QC, NB, NS

In 2012, rates of reported chronic HB for all age groups were higher among males than females, with the exception of the 25 to 29 age group, in which the reverse was found. Overall, the highest rates of reported cases of chronic HB in 2012 were observed among males in the 30 to 39 age group, followed by females in the 25 to 29 age group (25.9 and 25.6 per 100,000 respectively). In 2012 rates were higher among individuals between the ages of 20 and 59, with lower rates observed in both males and females of younger ( $<20$ years) and older $(>60$ years) age groups (Figure 4).

Figure 4. Rates of reported cases of chronic HB in Canada* by age group and sex, CNDSS, 2012

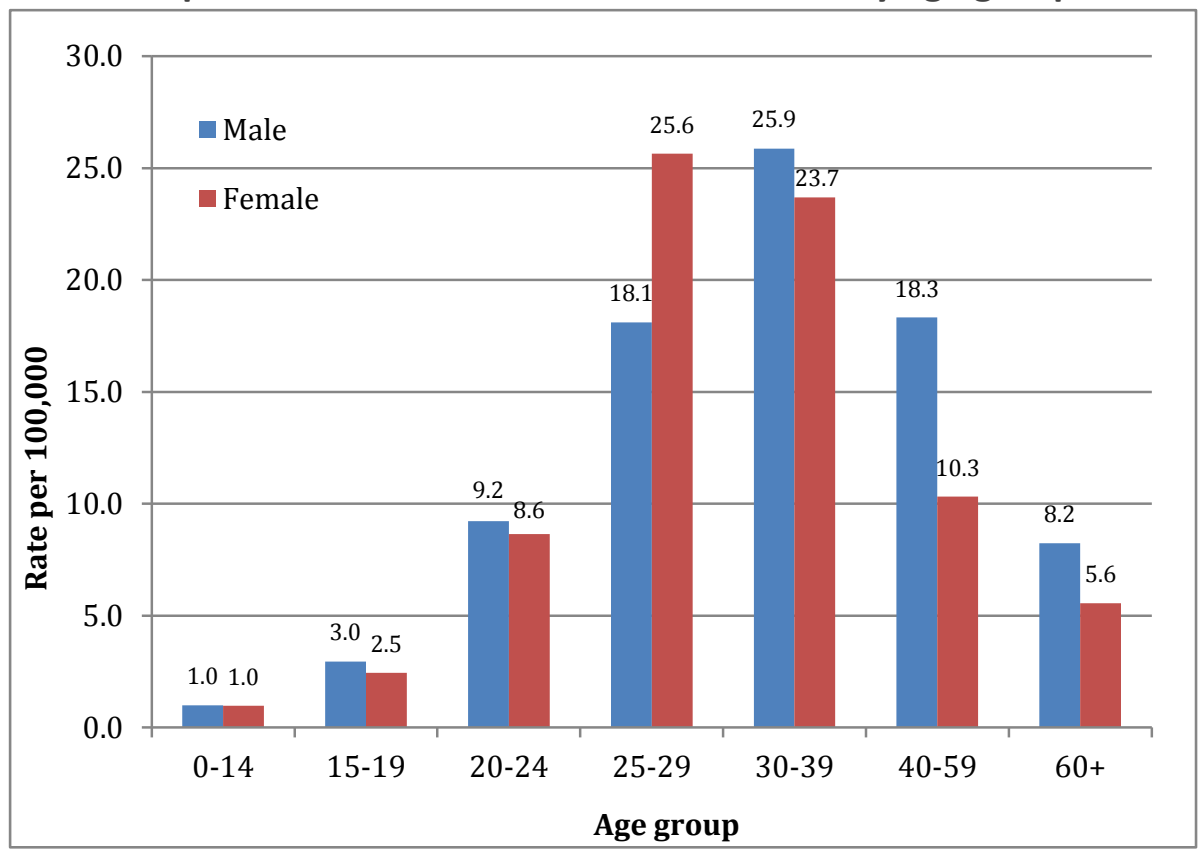


*Includes BC, AB, SK, MB, QC, NB, NS, YT

\section{Discussion}

This is the first time that trends in reported cases and corresponding rates of HB in Canada have been examined by acute and chronic infection using national surveillance data. It provides insights into general trends, based on the provinces and territories that were able to report data on HB infection status to the CNDSS.

Acute HB cases offer valuable insight into current transmission trends and patterns in Canada. There was a $45 \%$ decrease in the rate of reported acute hepatitis cases in Canada over the reporting period, These low rates may be attributable to the implementation of routine HB immunization. Children who received HB vaccine in the early 1990s when routine HB immunization programs first started have now reached adulthood. As an increasing proportion of the Canadian population is covered by HB immunization, it is reasonable to expect continued decreases in acute HB rates.

It is not surprising that similar decreases were not seen in the number of chronic HB infections. Although chronic HB infection rates are generally low in the general Canadian population, high rates have been documented among people born outside Canada. It has been estimated that approximately $4 \%$ of immigrants in Canada are chronically infected with HB (13).

Declining rates of acute HB have been similarly observed in countries with comparable population structure, health status and public health infrastructure, as evidenced by data from routine and/or enhanced surveillance (14-17). With respect to chronic HB infection, Canadian rates were considerably lower than those observed in other countries, though cross-country comparison is difficult because of differences in reporting practices $(14,16)$.

\section{Limitations}

These findings need to be interpreted in light of several limitations. First, the data are based on the number of reported cases; changes in provincial/territorial diagnostic and reporting practices may affect trends. By limiting our analysis to time periods and jurisdictions for which reporting was consistent, we have attempted to mitigate these effects. Additionally, when rates are based on small numbers of reported cases, they are more prone to fluctuations over time.

Second, the observed rates may be an underestimate of true HB incidence, possibly as a result of underdiagnoses of subclinical or mildly symptomatic cases. According to results from the Community Health Measures Survey, only $46 \%$ of Canadian respondents who tested positive for a current HB infection reported that they had been diagnosed with HB (18). Cases of chronic HB in Canada reported here are known to be an underestimate because of the unavailability of chronic HB data from Ontario, where a significant proportion of the Canadian population reside, many of whom are immigrants from countries where HB is endemic (19). A recent assessment of liver disease estimated that approximately $50 \%$ of individuals with chronic HB in Canada reside in Ontario (20).

Third, longer-term trends of chronic HB in Canada cannot be examined, as only a few provinces and territories reported chronic HB data before 2009.

Fourth, as only acute and chronic HB cases are analyzed in detail in this report, the findings are not inclusive of all HB cases reported to the CNDSS; infection status cannot be determined for all HB cases, In 2012, 737 (22.6\%) of the 3,262 HB cases reported nationally did not specify infection status. These unspecified cases could contribute to higher rates for either acute or chronic cases if infection status could be determined.

Finally, the data are limited to analysis by age, sex and infection status. At this time, there are no additional data elements in the CDNSS that could explain observed trends. Consequently, it is not clear what proportion of reported HB infections is due to importation of cases through immigration from endemic countries, injection drug use or high-risk sexual practices. 


\section{Conclusion}

From a public health planning and policy development perspective, it is important to identify populations disproportionately affected by HB as well as the factors associated with transmission of infection. Surveillance, supported by research that examines factors affecting observed trends, could contribute to the development and amelioration of tailored HB interventions in Canada.

\section{References}

(1) World Health Organization. Hepatitis B vaccines. Weekly Epidemiological Record 2009;84(40):405420.

(2) Tang CM, Yau TO, Yu J. Management of chronic hepatitis B infection: Current treatment guidelines, challenges, and new developments. World J Gastroenterol 2014 May 28;20(20):6262-6278.

(3) Heymann D editor. Control of communicable diseases manual. 19th ed. United States of America: American Public Health Association; 2008.

(4) Statistics Canada, Canadian Vital Statistics, Death Database. CANSIM Table 102-0521. Deaths, by cause, Chapter I: Certain infectious and parasitic diseases (A00 to B99), age group and sex, Canada. 2014.http://www5.statcan.gc.ca/cansim/a26?lang=eng\&retrLang=eng\&id=1020521\&pattern=death\&tabMode $=$ dataTable\&srchLan=-1\&p1=1\&p2=-1. Accessed June, 2014.

(5) National Advisory Committee on Immunization (NACl). Canadian national immunization report: Program update. Paediatr Child Health 1999;4(Suppl C):30C.

(6) Public Health Agency of Canada. Primary Care Management of Hepatitis B - Quick Reference. 2013.

(7) Public Health Agency of Canada. Canadian immunization guide: Part 4 active vaccines -Hepatitis $B$ vaccine. 2012. http://www.phac-aspc.gc.ca/publicat/cig-gci/p04-hepb-eng.php. Accessed July, 2013.

(8) Health Canada. Canadian immunization guide - sixth edition. 2002.

(9) Laroche J, Frescura A, Belzak L. Results from the 2006 and 2009 Childhood National Immunization Coverage Surveys. Canadian Immunization Conference, Québec City, QC 2010.

(10) Public Health Agency of Canada. Vaccine coverage in Canadian children: Results from the 2011 Public Childhood National Immunization Coverage survey. 2014. http://www.phac-aspc.gc.ca/im/nicsenva/vccc-cvec-eng.php. Accessed May, 2014.

(11) Public Health Agency of Canada. Vaccine coverage amongst adult Canadians: Results from the 2012 adult National Immunization Coverage (aNIC) survey. 2014. http://www.phac-aspc.gc.ca/im/nicsenva/vcac-cvac-eng.php. Accessed May, 2014.

(12) Public Health Agency of Canada. Supplement - case definitions for communicable diseases under national surveillance - 2009. Canada Communicable Disease Report 2009;35(Supplement 2).

(13) Greenway C, Narasiah L, Plourde P, Ueffing E, Pottie K, Deschenes MH, Wong DKH, Kuhn S, Heathcote JE; for the Canadian Collaboration for Immigrant and Refugee Health. Appendix 5: Hepatitis B: Evidence review for newly arriving immigrants and refugees. Canadian Medical Association Journal 2011;183(12).

(14) Centers for Disease Control and Prevention. Viral hepatitis surveillance - United States, 2011. 2011.

(15) Health Protection Agency. Acute hepatitis B (England): annual report for 2011. Health Protection Report 2012;6(34). 
(16) Australian Government Department of Health. Australia's notifiable disease status, 2011: Annual report of the National Notifiable Diseases Surveillance System. 2013.

(17) Centers for Disease Control and Prevention. Viral hepatitis statistics and surveillance: table $1 \mathrm{~b}$. Incidence per 100,000 population of acute symptomatic hepatitis A by state/area and year - United States, 1996-2008. 2010. http://www.cdc.gov/hepatitis/Statistics/2008Surveillance/Table1b.htm. Accessed July, 2013.

(18) Rotermann M, Langlois K, Andonov A, Trubnikov M. Seroprevalence of hepatitis B and C virus infections: Results from the 2007 to 2009 and 2009 to 2011 Canadian Health Measures Survey. Health Rep 2013;24(11):3-13.

(19) Human Resources and Skills Development Canada. Canadians in context - geographic distribution. 2013. http://www4.hrsdc.gc.ca/.3ndic.1t.4r@-eng.jsp?iid=34\&bw=1. Accessed August 2013.

(20) Canadian Liver Foundation. Liver disease in Canada: A crisis in the making. 2013.

\section{Acknowledgements}

The authors would like to acknowledge the participation of health care providers, public health officials and laboratories, and the provinces and territories involved in the routine reporting of $\mathrm{HB}$.

We would also like to acknowledge and thank Rachel MacLean for her contribution to HB surveillance at the Public Health Agency of Canada.

\section{Conflicts of interest}

No conflicts of interest to declare. 